\title{
Unified SISO Loop Gain Modeling, Measurement, and Stability Analysis of Three- Phase Voltage Source Converters
}

\author{
Jianheng Lin, Mei Su, Member, IEEE, Yao Sun, Member, IEEE, \\ Shiming Xie, Wenjing Xiong, and Xing Li
}

\begin{abstract}
Frequency-domain modeling is an effective technique in the dynamic analysis of power electronic convertersbased power systems. In this paper, a unified single-input singleoutput (SISO) loop gain modeling for the three-phase grid-tied VSCs under both symmetric and asymmetric ac grids is presented, which facilitates the physical measurement and stability analysis. Based on the linear-time-periodic (LTP) modeling technique, the harmonic admittance model of the three-phase grid-tied VSC is developed in the stationary $(\alpha \beta)$-frame. Instead of the transfer function matrix, the frequency-coupling effects are modeled by the transfer function vector, which simplifies the modeling process. According to the idea of mathematical induction, a two-by-two recursive admittance matrix (RAM) model that can accurately capture the coupling dynamics introduced by the power grid is derived. The RAM has an analytical form and is easy to include harmonic coupling components of arbitrary order. Furthermore, the RAM is converted to its equivalent SISO models following the concept of loop gain. The system stability is thus assessed by the SISO stability criteria (e.g., Nyquist stability criterion). In addition, the loop gain allows the traditional SISO perturbation and measurement scheme to be used for detecting the stability margin information. Finally, simulation results verify the feasibility and correctness of the theoretical analysis presented above.
\end{abstract}

Index Terms-Frequency-domain modeling, loop gain, frequency-coupling effect, measurement, voltage source converter.

\section{INTRODUCTION}

$\mathrm{N}$ OWADAYS, voltage source converters (VSCs) have been widely used in power grids, such as distributed generation and flexible power transmission [1], [2]. The dynamic interactions between power converters and passive components (e.g., power filter and grid impedance) may lead to some unstable phenomena, which would damage the safe operation of power systems [3]-[5]. Therefore, it is of great importance to analyze the stability of the converter-grid interactive systems.

To address the stability challenges in VSC-grid systems, the analytical impedance-based approach is an attractive solution. A classic approach to model VSCs is to utilize the Park transformation to transform the time-periodic operating trajectory as the time-invariant point in the $d q$-frame, and then linearize the system to obtain a linear time-invariant (LTI) multi-input multi-output (MIMO) impedance model [6]. On this basis, many detailed impedance models including different factors are formulated, e.g. constant power load [7] and PLL [8], [9]. In [10]-[12], variants of the $d q$-impedance modeling technique are comprehensively overviewed. However, the $d q$ - frame modeling relies on the assumption that the VSC system is three-phase balanced, thereby limiting its application in asymmetric ac grids.

As for VSCs connecting with asymmetric ac grids, the $d q$ frame modeling is not suitable since the time-periodic operating trajectories cannot be transformed into time-invariant operating points by Park transformation. After performing linearization, the resulting VSC model is characterized by linear timeperiodic (LTP). Unlike the LTI model, translating the LTP model into the frequency domain is no longer straightforward due to the presence of frequency couplings [13], [14]. Thus, many research efforts have been made to address timeperiodicity challenges.

In general, there have been three general multi-frequency modeling methods that can deal with time-periodic systems. The first approach is dynamic phasor modeling [15], [16]. Initially, dynamic-phasor-based modeling is mainly used for efficient simulations [17]. After being validated, numerous works have been focused on stability analysis based on dynamic phasor models. In dynamic phasor modeling, different coordinate transformations (e.g., multiple Park transformation [18]) can be embedded for model simplification. The harmonic linearization method behind the idea of describing function is the second approach [19]. When the frequency coupling effect (FCE) is not neglectable (strong asymmetry of the controller and grid condition, extremely weak grid), this method was confirmed to be inaccurate [20], [21]. Therefore, some improved versions of harmonic linearization have been proposed in [22] and [23], which incorporate the cross-coupling terms into modeling. Recently, the harmonic transfer function (HTF) modeling established on the Floquet theory has gained popularity, which is the third approach [13], [24]. Unlike the first two methods, the HTF method directly linearizes the system around the time-periodic trajectories [4], [25]. Note that the information of the Toeplitz matrix is redundant in describing the FCEs, a general LTP modeling is further proposed in [26] that improves model compactness.

The dynamic phasor models under unbalanced grid voltages have been studied in [27] and [28]. However, the dynamic phasor models are mostly formulated in the time domain so that the explicit frequency-domain representations need to be further derived. In contrast, the harmonic linearization and the HTF approach directly establish the model in the frequency domain. Based on the harmonic linearization technique, the sequence impedance models of the VSC under asymmetric ac 
grids are obtained in [29] and [30]. The convolution operation is required that increases the modeling complexity. Besides, in [29], it is improper that replacing unbalanced grid voltages with asymmetric grid impedances because the grid impedance would introduce additional closed-loop frequency coupling dynamics. The use of HTF for VSC modeling in unbalanced grids has been recently reported in [31] and [32]. Although the frequencycoupling dynamics inside the converter have been fully studied in the works, the inherent cross-coupling between the converter and the power grid is not well studied.

At present, the existing impedance models of grid-tied VSC systems considering the asymmetric ac grids are all represented by impedance matrices [29]-[32]. In this regard, the generalized Nyquist criterion (GNC) should be adopted for stability analysis and multiple perturbations are required in the frequency-sweeping. Therefore, finding the analytical SISO equivalents of grid-tied VSC systems is attractive due to their simplicity and convenience for physical interpretation. The model reduction techniques under balanced grids have been discussed in [10] and [33]. In [10], following the definition of impedance, the SISO equivalent grid/converter impedances are separately obtained through algebraic calculation. Hereafter, the two SISO equivalent impedances are further simplified to a SISO loop impedance in [33], which is the characteristic equation of the system. Overall, the SISO equivalent modeling technique for asymmetric ac grid analysis is still missing here. On the other hand, it was demonstrated in the single-phase system that there is low-order harmonic coupling between the converter and the power grid, and the coupling becomes stronger with the increase of the grid impedance [26]. However, the converter-grid coupling analysis in the three-phase VSC system is not well studied so far. The above reasons motivate the authors to do the work of this paper.

To explore the modeling, measurement, and stability analysis of VSCs, this article proposes a unified SISO loop gain modeling for the three-phase grid-tied VSC system under both symmetric and asymmetric ac grids. The main contributions of this paper are summarized as:

1) A simple and general harmonic admittance modeling method of three-phase VSC is presented, which accurately captures the FCE inside the converter.

2) Incorporating the converter-grid coupling, a two-by-two recursive admittance matrix (RAM) model is proposed. It is illustrated that the VSC admittance varies along with the grid admittance in the low-frequency regions.

3) Based on the concept of loop gain, an analytical and compact SISO equivalent model is derived from the RAM. The existing measurement schemes and SISO stability analysis criteria can be directly applied to the developed loop gain model.

The rest of this paper is organized as follows. In Section II, the system configuration is described and the frequency coupling effect is briefly introduced. Section III presents the harmonic admittance modeling under a general grid condition. In Section IV, the SISO loop gain modeling is presented. In Section V, model validation is provided and the system stability analysis is conducted under asymmetric ac grids. Finally, Section VI concludes this paper.

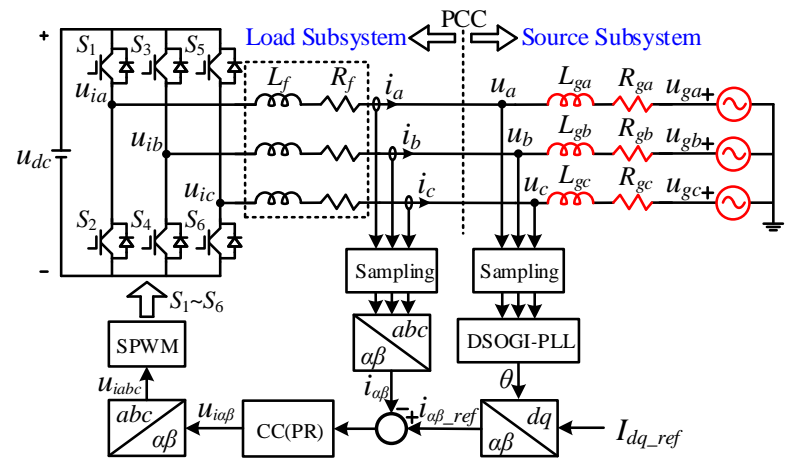

Fig. 1. Schematic of a three-phase VSC for grid-connected applications.

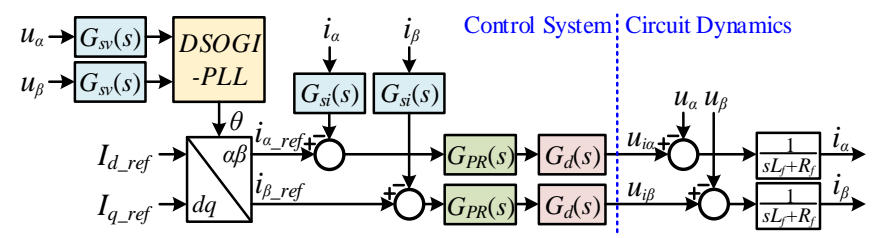

Fig. 2. The simplified block diagram of the three-phase VSC system.

\section{THREE-PHASE GRID-TIED VSC SYSTEM}

\section{A. System Configuration}

The studied three-phase grid-tied VSC and its control system are depicted in Fig. 1. It is comprised of a typical two-level voltage source converter, symmetric $L$-type filter $\left(L_{f} / R_{f}\right)$, and a Thevenin equivalent grid. The dc-link voltage $u_{d c}$ is assumed to be constant. Phase voltages at the point of common coupling (PCC) are denoted as $u_{a}, u_{b}$, and $u_{c}$, while phase currents as $i_{a}$, $i_{b}$, and $i_{c} . L_{g a} / R_{g a}, L_{g b} / R_{g b}$, and $L_{g c} / R_{g c}$ denote the per-phase equivalent grid impedance, respectively. The phase grid voltages are $u_{g a}, u_{g b}$, and $u_{g c}$. A general grid condition is considered in this paper that the grid impedances and the grid voltages are not necessarily balanced.

Without loss of generality, only the current controller and phase-locked loop (PLL) are considered for simplicity, while the same conclusions with other control methods are achieved in the same way.

In the control system, the dual second-order generalized integrator phase-locked loop (DSOGI-PLL) is used due to its superior adaptivity to the fault grids [34]. The DSOGI-PLL tracks the phase $\theta$ of the positive-sequence PCC voltage, avoiding the phase oscillation caused by negative-sequence voltage. The phase currents are regulated by a proportionalresonant (PR) current controller (CC). The current references ( $I_{d_{\_} r e f}$ and $\left.I_{q_{-} r e f}\right)$ are assumed constant.

Fig. 2 depicts the simplified block diagram of the three-phase VSC system in the stationary frame. The sampling delay is modeled as

$$
\begin{aligned}
G_{s i}(s) & =\frac{1}{1+s / \omega_{i}} \\
G_{s v}(s) & =\frac{1}{1+s / \omega_{v}}
\end{aligned}
$$

where $\omega_{i}$ and $\omega_{v}$ are the cutoff frequency of anti-aliasing filters for current and voltage measurement, respectively. 


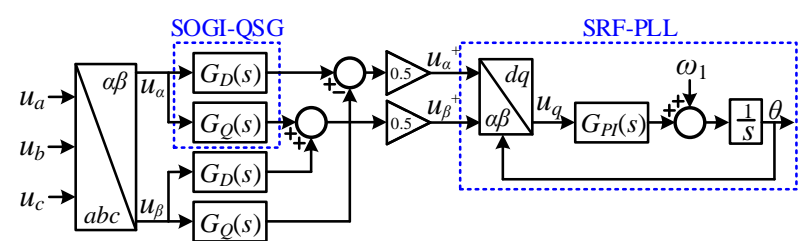

Fig. 3. Block diagram of the DSOGI-PLL.

The PR regulator is given as

$$
G_{P R}(s)=k_{p c}+\frac{k_{r c} s}{s^{2}+\omega_{1}^{2}}
$$

where $\omega_{1}$ is the angular frequency of the grid, and $k_{p c}$ and $k_{r c}$ are the PR controller parameters.

Zero-order hold $(\mathrm{ZOH})$ effects and calculation delays are taken into consideration as

$$
G_{d}(s)=e^{-s T_{s}} \frac{1-e^{-s T_{s}}}{s T_{s}}
$$

where $T_{s}$ is the control period.

Fig. 3 draws the block diagram of DSOGI-PLL, which consists of two second-order generalized integrator quadraturesignals generators (SOGI-QSGs) and a synchronous reference frame (SRF)-PLL [34]. The SOGI-QSG and the proportionalintegral (PI) regulator are given as

$$
\begin{gathered}
G_{D}(s)=\frac{2 \xi \omega_{1} s}{s^{2}+2 \xi \omega_{1} s+\omega_{1}^{2}} \\
G_{Q}(s)=\frac{2 \xi \omega_{1}^{2}}{s^{2}+2 \xi \omega_{1} s+\omega_{1}^{2}} \\
G_{P I}(s)=k_{p p}+\frac{k_{i p}}{s}
\end{gathered}
$$

where $\xi$ is the damping ratio, $k_{p p}$ and $k_{i p}$ are the PI controller parameters.

\section{B. Frequency-Coupling Effect In VSC}

To establish the impedance model or measure the ac-side impedance of the VSC system, the perturbation voltage or current is usually injected into the ac system [10]. However, it was observed that the injected perturbation voltage/current not only generates the current/voltage at the same frequency but also the sideband components whose frequencies are shifted with twice the fundamental frequency, which is called the frequency-coupling phenomenon [4], [5].

To better visualize the frequency-coupling phenomenon, the typical time-domain waveform is illustrated in Fig. 4, where the PCC phase voltage $u_{a}$ is intentionally perturbed at the frequency $70 \mathrm{~Hz}$ (3\% of the fundamental). The a-phase current $i_{a}$ is taken as an example for spectral analysis and the same conclusions are achieved in other phases. Fig. 4(b) shows that the phase current is composed of the corresponding component $(70 \mathrm{~Hz})$ and the coupling components $(30 \mathrm{~Hz}, 170 \mathrm{~Hz})$.

As reported in [20]-[26], [29]-[32], the neglect of FCE in the modeling would degrade the model accuracy. Moreover, due to the FCE, additional sideband loops are formed through the grid impedance, which strengthens the converter-grid coupling [26]. In summary, it is necessary to consider the FCE in the system modeling.

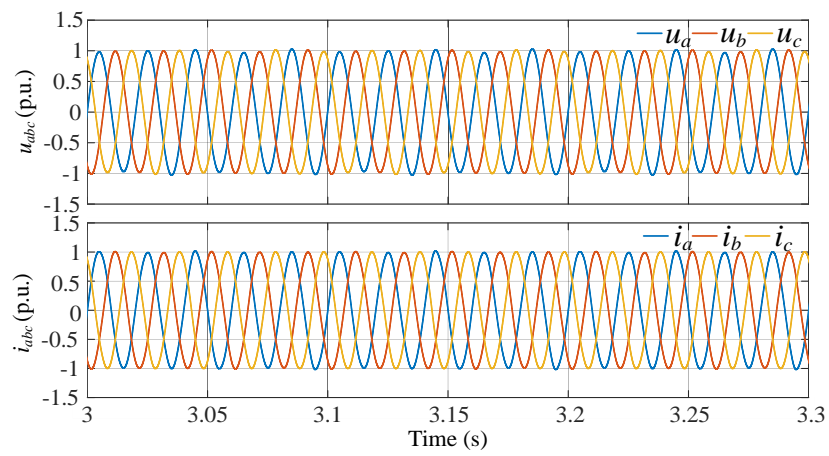

(a) Time-domain waveforms

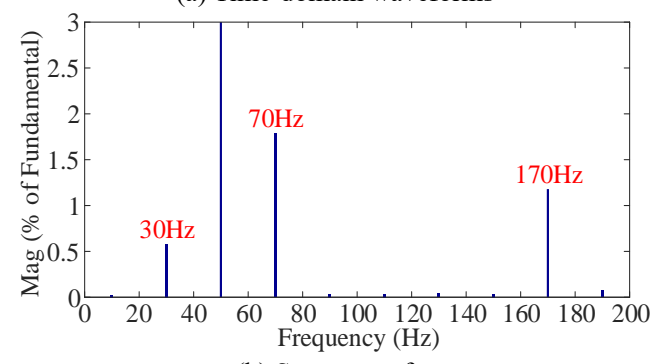

(b) Spectrum of $i_{a}$

Fig. 4. Illustration of the FCE phenomenon when the PCC voltage is perturbed at the frequency $70 \mathrm{~Hz}$.

\section{HARMONIC ADMITTANCE MODELING}

In this paper, the three-phase VSC is directly modeled in the stationary frame. Compared with the $d q$-frame model, no additional transformation is needed so that the $\alpha \beta$-frame model is of clear physical insight and facilitates the physical measurement.

\section{A. DSOGI-PLL Modeling}

According to the structure of the DSOGI-PLL, the $q$ axis voltage $u_{q}$ is obtained as

$$
u_{q}=-\sin (\theta) u_{\alpha}^{+}+\cos (\theta) u_{\beta}^{+}
$$

where $u_{\alpha}{ }^{+}$and $u_{\beta}{ }^{+}$are the positive-sequence component of $\alpha \beta$ frame voltages $u_{\alpha}$ and $u_{\beta}$, respectively.

Perturbing the variables in the time-domain, the linearized form of (8) is derived as

$$
\tilde{u}_{q}=-\sin (\bar{\theta}) \tilde{u}_{\alpha}^{+}+\cos (\bar{\theta}) \tilde{u}_{\beta}^{+}-\cos (\bar{\theta}) \bar{u}_{\alpha}^{+} \tilde{\theta}-\sin (\bar{\theta}) \bar{u}_{\beta}^{+} \tilde{\theta}
$$

where the superscript ' $\sim$ ' and '-' represent the small-signal and steady-state quantities, respectively. The steady-state quantities are given by $\bar{\theta}=\omega_{1} t, \bar{u}_{\alpha}{ }^{+}=V_{p} \cos \left(\omega_{1} t\right)$, and $\bar{u}_{\beta}{ }^{+}=V_{p} \sin \left(\omega_{1} t\right)$, where $V_{p}$ is the magnitude of the positive-sequence voltage component at the fundamental frequency.

Substituting the steady-state quantities into (9) gives

$$
\tilde{u}_{q}=-\sin \left(\omega_{1} t\right) \tilde{u}_{\alpha}^{+}+\cos \left(\omega_{1} t\right) \tilde{u}_{\beta}^{+}-V_{p} \tilde{\theta}
$$

Then, according to [26], the frequency domain expression of (10) can be obtained as

$$
\begin{aligned}
\tilde{u}_{q}(s) & =\frac{j}{2}\left[\tilde{u}_{\alpha}^{+}\left(s-j \omega_{1}\right)-\tilde{u}_{\alpha}^{+}\left(s+j \omega_{1}\right)\right] \\
& +\frac{1}{2}\left[\tilde{u}_{\beta}^{+}\left(s-j \omega_{1}\right)+\tilde{u}_{\beta}^{+}\left(s+j \omega_{1}\right)\right]-V_{p} \tilde{\theta}(s)
\end{aligned}
$$

Based on the DSOGI-PLL diagram, the following relationships hold 


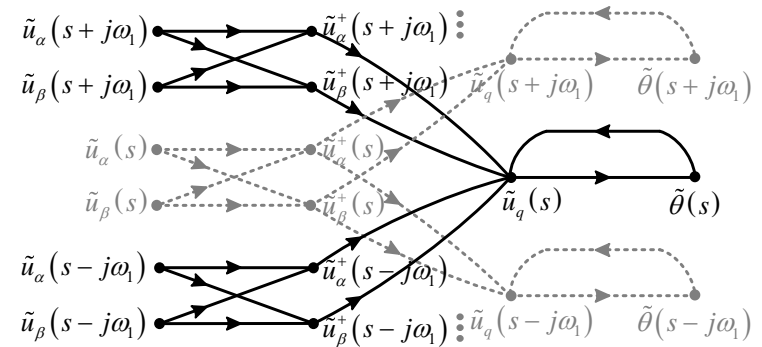

Fig. 5. Harmonic signal-flow graph of the DSOGI-PLL.

$$
\begin{gathered}
\tilde{u}_{\alpha}^{+}(s)=\frac{G_{s v}(s)}{2}\left[G_{D}(s) \tilde{u}_{\alpha}(s)-G_{Q}(s) \tilde{u}_{\beta}(s)\right] \\
\tilde{u}_{\beta}^{+}(s)=\frac{G_{s v}(s)}{2}\left[G_{D}(s) \tilde{u}_{\beta}(s)+G_{Q}(s) \tilde{u}_{\alpha}(s)\right] \\
\tilde{\theta}(s)=G_{P L L}(s) \tilde{u}_{q}(s)
\end{gathered}
$$

where $G_{P L L}(s)=G_{P I}(s) / s$.

Substituting (12)-(14) into (11) gives (15), shown at the bottom of the page. The expression of $T_{P L L}(s)$ is given as

$$
T_{P L L}(s)=\frac{1}{2} \frac{G_{P L L}(s)}{s+V_{p} G_{P L L}(s)}
$$

As denoted by (15), it clearly shows that the perturbed PLL output $\tilde{\theta}$ at a given frequency $\omega$ is related to the PCC voltages $\tilde{u}_{\alpha}$ and $\tilde{u}_{\beta}$ at the two coupled frequencies $\omega \pm \omega_{1}$. Fig. 5 plots the harmonic signal-flow graph of DSOGI-PLL, where the transfer function gain is omitted for brevity.

\section{B. Harmonic Admittance Modeling}

The inverter current references are expressed as

$$
\left[\begin{array}{l}
i_{\alpha_{-} \text {ref }} \\
i_{\beta_{-} \text {ref }}
\end{array}\right]=\left[\begin{array}{cc}
\cos (\theta) & -\sin (\theta) \\
\sin (\theta) & \cos (\theta)
\end{array}\right]\left[\begin{array}{l}
I_{d_{-}} \text {ref } \\
I_{q_{-} \text {ref }}
\end{array}\right]
$$

Following the same steps as before, the linearized model of (17) in the frequency domain can be derived as

$$
\left[\begin{array}{c}
\tilde{i}_{\alpha_{-} r e f}(s) \\
\tilde{i}_{\beta_{-} r e f}(s)
\end{array}\right]=\left[\begin{array}{cc}
k_{1} & k_{2} \\
-j k_{1} & j k_{2}
\end{array}\right]\left[\begin{array}{l}
\tilde{\theta}\left(s-j \omega_{s}\right) \\
\tilde{\theta}\left(s+j \omega_{s}\right)
\end{array}\right]
$$

where $k_{1}=\left(-I_{q_{-} r e f}+j I_{d_{-} r e f}\right) / 2$ and $k_{2}=-\left(I_{q_{-} r e f}+j I_{d_{-} r e f}\right) / 2$.

For the grid side, the closed-loop response of the current control loop can be given by

$$
\begin{aligned}
& \tilde{\theta}(s)=\frac{1}{2} T_{P L L}(s) G_{s v}\left(s-j \omega_{1}\right)\left\{\left[j G_{D}\left(s-j \omega_{1}\right)+G_{Q}\left(s-j \omega_{1}\right)\right] \tilde{u}_{\alpha}^{+}\left(s-j \omega_{1}\right)+\left[G_{D}\left(s-j \omega_{1}\right)-j G_{Q}\left(s-j \omega_{1}\right)\right] \tilde{u}_{\beta}^{+}\left(s-j \omega_{1}\right)\right\} \\
& +\frac{1}{2} T_{P L L}(s) G_{s v}\left(s+j \omega_{1}\right)\left\{\left[G_{Q}\left(s+j \omega_{1}\right)-j G_{D}\left(s+j \omega_{1}\right)\right] \tilde{u}_{\alpha}^{+}\left(s+j \omega_{1}\right)+\left[j G_{Q}\left(s+j \omega_{1}\right)+G_{D}\left(s+j \omega_{1}\right)\right] \tilde{u}_{\beta}^{+}\left(s+j \omega_{1}\right)\right\} \\
& \boldsymbol{Y}_{p}(s)=\frac{k_{1}}{2} T_{P L L}\left(s+j \omega_{1}\right) G_{p l a n t}\left(s+j 2 \omega_{1}\right) G_{s v}(s)\left[\begin{array}{cc}
G_{Q}(s)+j G_{D}(s) & G_{D}(s)-j G_{Q}(s) \\
-j G_{Q}(s)+G_{D}(s) & -j G_{D}(s)-G_{Q}(s)
\end{array}\right] \\
& \boldsymbol{Y}_{0}(s)=\frac{1}{2} G_{p l a n t}(s) G_{s v}(s)\left\{\begin{array}{l}
k_{1} T_{P L L}\left(s-j \omega_{1}\right)\left[\begin{array}{cc}
G_{Q}(s)-j G_{D}(s) & G_{D}(s)+j G_{Q}(s) \\
-j G_{Q}(s)-G_{D}(s) & -j G_{D}(s)+G_{Q}(s)
\end{array}\right] \\
+k_{2} T_{P L L}\left(s+j \omega_{1}\right)\left[\begin{array}{ll}
G_{Q}(s)+j G_{D}(s) & G_{D}(s)-j G_{Q}(s) \\
j G_{Q}(s)-G_{D}(s) & j G_{D}(s)+G_{Q}(s)
\end{array}\right]
\end{array}\right\}-\left[\begin{array}{cc}
Y_{i n v_{-} o}(s) & 0 \\
0 & Y_{i n v_{-} o}(s)
\end{array}\right] \\
& \boldsymbol{Y}_{n}(s)=\frac{k_{2}}{2} T_{P L L}\left(s-j \omega_{1}\right) G_{p l a n t}\left(s-j 2 \omega_{1}\right) G_{s v}(s)\left[\begin{array}{ll}
G_{Q}(s)-j G_{D}(s) & G_{D}(s)+j G_{Q}(s) \\
j G_{Q}(s)+G_{D}(s) & j G_{D}(s)-G_{Q}(s)
\end{array}\right]
\end{aligned}
$$




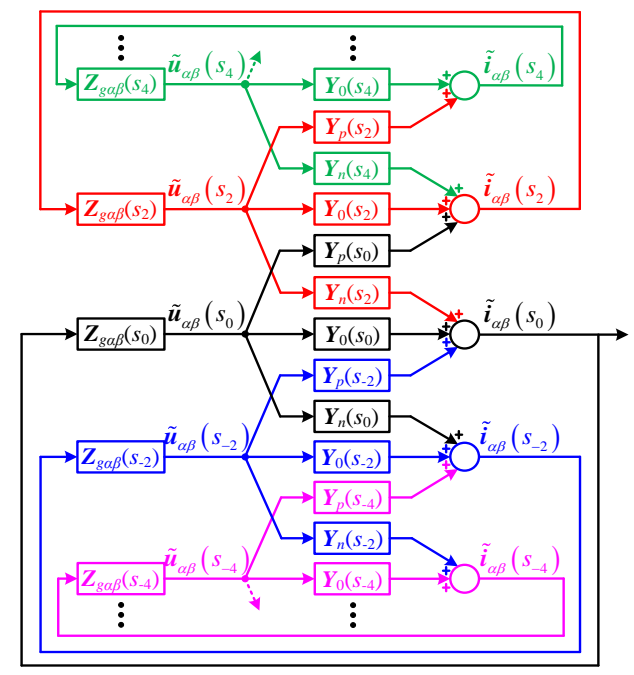

Fig. 7. Multi-frequency diagram of the three-phase grid-connected VSC considering the grid impedance.

From (23), it is shown that the perturbed grid current $\tilde{\boldsymbol{i}}_{\alpha \beta}$ at a given frequency $\omega$ is related to the PCC voltage $\tilde{\boldsymbol{u}}_{\alpha \beta}$ at the three coupled frequencies $\omega$ and $\omega \pm 2 \omega_{1}$, which agrees with Fig. 4. Moreover, it is found that the negative sequence voltage $V_{n}$ caused by PCC voltage imbalance has no impact on the converter harmonic admittance due to the use of DSOGI-PLL.

Remark: Many modeling methods can lead to accurate models, but the modeling procedures and the resulting models are different. On one hand, the describing-function-based methods require the concept of signal injection [19]-[22], [29], [30], where the form of perturbations needs to be defined empirically and complex convolution operation is required. In the HTF-based modeling methods [25], [31]-[32], the use of large-dimensional matrix operation degrades the model analyticity and increases the computation effort. In contrast, the proposed harmonic admittance modeling does not need perturbation injection and large-dimensional matrix operation, therefore reducing the modeling complexity.

On the other hand, the harmonic admittance model in (23) is represented by the real-valued vector. Compared with the complex-valued models [12] and [32], the real-valued models have the following characteristics:

1) There is no need to perform additional transformations.

2) The complex conjugation is avoided.

3) The FCE is intuitively interpreted in the original frame.

\section{SISO LOOP GAIN MODELING OF THE GRID-TIED VSC}

\section{A. Recursive Admittance Matrix Modeling}

The developed VSC harmonic admittance model in (23) is physically intuitive but it is not straightforward to be used for stability analysis due to the cross-coupling terms. The most simplified admittance model derived from (23) is $-\boldsymbol{Y}_{0}(s)$. However, the simplified model may lead to inaccurate analysis results and hides the converter-grid coupling phenomenon. Therefore, the frequency couplings should be considered.

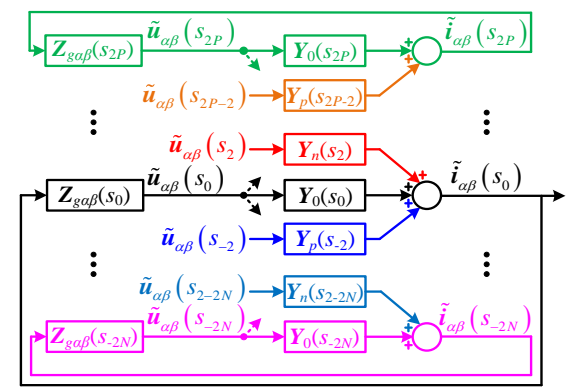

Fig. 8. Truncated multi-frequency diagram with $P$ positive loops and $N$ negative loops.

Considering the sideband loops caused by the FCE, the multi-frequency diagram of the three-phase grid-connected VSC is drawn as Fig. 7, where $s_{k}$ is defined as $s+j k \omega_{1}(k \in \mathbb{Z})$. It is observed that the converter is coupled with the grid since the additional loops caused by the FCE are closed through the grid impedance. Undoubtedly, the converter-grid coupling will have an impact on the VSC admittance, which has not been fully studied in the existing literature.

As shown in Fig. 7, the sideband loops are the translated copies of the fundamental loop (denoted by the black line) with frequency shifting $\pm k \omega_{1}$. Moreover, the order of harmonic coupling extends to infinite in the presence of the grid impedance. In this case, the complete admittance should be represented by an infinite-dimensional matrix conceptually [31], [32], which is rather complicated to analyze and implement. Thus, from the view of analysis and validation, finding the lowdimensional equivalent admittance model is appealing [26].

According to Fig. 7, the relationship between $\tilde{\boldsymbol{i}}_{\alpha \beta}$ and $\tilde{\boldsymbol{u}}_{\alpha \beta}$ in different frequency domains can be consistently expressed as

$$
\begin{aligned}
\tilde{\boldsymbol{i}}_{\alpha \beta}\left(s_{K}\right) & =\boldsymbol{Y}_{p}\left(s_{K-2}\right) \tilde{\boldsymbol{u}}_{\alpha \beta}\left(s_{K-2}\right)+\boldsymbol{Y}_{0}\left(s_{K}\right) \tilde{\boldsymbol{u}}_{\alpha \beta}\left(s_{K}\right) \\
& +\boldsymbol{Y}_{n}\left(s_{K+2}\right) \tilde{\boldsymbol{u}}_{\alpha \beta}\left(s_{K+2}\right)
\end{aligned}
$$

where $K \in \mathbb{Z}$.

In addition, due to the existence of grid impedance, it is established that

$$
\begin{aligned}
\tilde{\boldsymbol{i}}_{\alpha \beta}\left(s_{K}\right) & =\left[\begin{array}{ccc}
Y_{g \alpha \alpha}\left(s_{K}\right) & Y_{g \alpha \beta}\left(s_{K}\right) \\
Y_{g \beta \alpha}\left(s_{K}\right) & Y_{g \beta \beta}\left(s_{K}\right)
\end{array}\right] \tilde{\boldsymbol{u}}_{\alpha \beta}\left(s_{K}\right) \\
& =\boldsymbol{Y}_{g \alpha \beta}\left(s_{K}\right) \tilde{\boldsymbol{u}}_{\alpha \beta}\left(s_{K}\right)
\end{aligned}
$$

where $K \in \mathbb{Z}$ and $\boldsymbol{Y}_{g \alpha \beta}(s)=\boldsymbol{Z}_{g a \beta}{ }^{-1}(s)$. The detailed derivation of the grid admittance model $\boldsymbol{Y}_{g \alpha \beta}(s)$ under a general grid condition is given in Appendix-A.

To obtain a reduced-order model, assume firstly that the multi-loop system is truncated to $P(P \in \mathbb{N})$ positive loops and $N(N \in \mathbb{N})$ negative loops as depicted in Fig. 8, the truncation conditions are expressed as

$$
\begin{cases}\tilde{\boldsymbol{u}}_{\alpha \beta}\left(s_{2 X}\right)=0, & X \geq P+1 \\ \tilde{\boldsymbol{u}}_{\alpha \beta}\left(s_{-2 Y}\right)=0, & Y \geq N+1\end{cases}
$$

where $X \in \mathbb{N}$ and $Y \in \mathbb{N}$.

The positive loop truncation is taken as an example to illustrate the recursive modeling technique. Based on the idea of mathematical induction, assume firstly that

$$
\boldsymbol{Y}_{n}\left(s_{2 X}\right) \tilde{\boldsymbol{u}}_{\alpha \beta}\left(s_{2 X}\right)=\boldsymbol{F}_{P}(P-(X-1)) \tilde{\boldsymbol{u}}_{\alpha \beta}\left(s_{2(X-1)}\right)
$$

where $1 \leq X \leq P+1, \boldsymbol{F}_{P}(P-(X-1))$ is a two-by-two mapping matrix 
from $\tilde{\boldsymbol{u}}_{\alpha \beta}\left(s_{2(X-1)}\right)$ to $\tilde{\boldsymbol{u}}_{\alpha \beta}\left(s_{2 X}\right)$ that needs to be determined, and $\boldsymbol{F}_{P}(0)=\mathbf{0}^{2 \times 2}$ due to $(29)$.

Next, replacing $K$ in (27) with $2 X$ gives

$$
\begin{aligned}
\tilde{\boldsymbol{i}}_{\alpha \beta}\left(s_{2 X}\right) & =\boldsymbol{Y}_{p}\left(s_{2(X-1)}\right) \tilde{\boldsymbol{u}}_{\alpha \beta}\left(s_{2(X-1)}\right)+\boldsymbol{Y}_{0}\left(s_{2 X}\right) \tilde{\boldsymbol{u}}_{\alpha \beta}\left(s_{2 X}\right) \\
& +\boldsymbol{Y}_{n}\left(s_{2(X+1)}\right) \tilde{\boldsymbol{u}}_{\alpha \beta}\left(s_{2(X+1)}\right)
\end{aligned}
$$

Combining (28) and (30) into (31) yields (32), shown at the bottom of the page. Comparing (30) and (32), $F_{P}(\bullet)$ can be solved as (33), shown at the bottom of the page.

In the same way, a recursive relationship for the negative loop truncation can be derived as

$$
\boldsymbol{Y}_{p}\left(s_{-2 Y}\right) \tilde{\boldsymbol{u}}_{\alpha \beta}\left(s_{-2 Y}\right)=\boldsymbol{F}_{N}(N-(Y-1)) \tilde{\boldsymbol{u}}_{\alpha \beta}\left(s_{-2(Y-1)}\right)
$$

where $1 \leq Y \leq N+1, \boldsymbol{F}_{N}(\cdot)$ is given as (35), shown at the bottom of the page.

Finally, combining (27), (30), and (34), a two-by-two RAM model $\boldsymbol{Y}(s)$ can be derived as

$$
-\tilde{\boldsymbol{i}}_{\alpha \beta}(s)=\underbrace{-\left[\boldsymbol{Y}_{0}(s)+\boldsymbol{F}_{N}(N)+\boldsymbol{F}_{P}(P)\right]}_{\boldsymbol{Y}(s)} \tilde{\boldsymbol{u}}_{\alpha \beta}(s)
$$

Compared with the existing three-phase VSC frequencydomain models [29]-[32], the advantages of the RAM model $\boldsymbol{Y}(s)$ are summarized as:

1) The RAM model has an analytical and compact expression, and it is easy to include harmonic coupling of arbitrary order.

2) The coupling effect between the converter and the power grid is embedded in the RAM model.

\section{B. SISO Loop Gain Modeling}

The RAM $Y(s)$ greatly simplifies the system model, but it is still a MIMO model with the order of two. To measure $\boldsymbol{Y}(s)$, cumbersome frequency-sweeping is required that perturbations should be injected twice at each frequency point. In addition, the GNC is needed for stability analysis, which is not intuitive due to the lack of traditional margin information. All these arguments point to the benefits of using a SISO model.

An intuitive SISO model is the one that only preserves the diagonal element of $-\boldsymbol{Y}_{0}(s)$ or $\boldsymbol{Y}(s)$. Although the diagonal element considers the FCE to a certain extent, its accuracy decreases as the asymmetry increases.

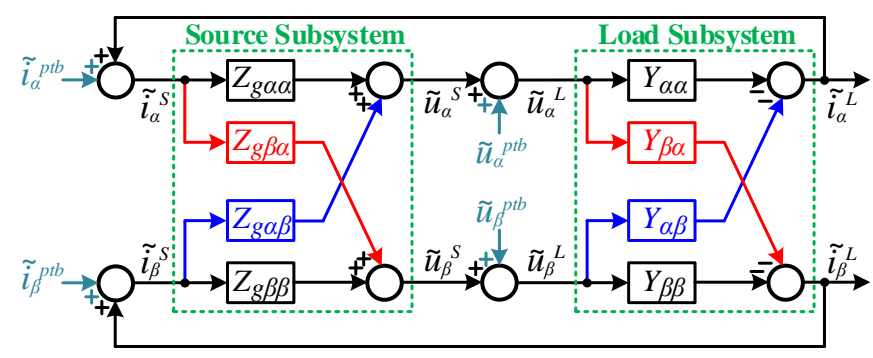

Fig. 9. Equivalent block diagram of the three-phase VSC in the stationary frame.

The concept of loop gain has been widely used in characterizing the dc/dc system [35]-[37]. The loop gain not only reflects the system stability margin but also can be directly measured through frequency sweeping. In this subsection, following the definition of loop gain, $\boldsymbol{Y}(s)$ is further simplified to its SISO equivalent model.

For the load (VSC) subsystem, its admittance model is

$$
-\left[\begin{array}{c}
\tilde{i}_{\alpha}^{L} \\
\tilde{i}_{\beta}^{L}
\end{array}\right]=\left[\begin{array}{cc}
Y_{\alpha \alpha} & Y_{\alpha \beta} \\
Y_{\beta \alpha} & Y_{\beta \beta}
\end{array}\right]\left[\begin{array}{c}
\tilde{u}_{\alpha}^{L} \\
\tilde{u}_{\beta}^{L}
\end{array}\right]
$$

For the source (grid) subsystem, its impedance model is

$$
\left[\begin{array}{c}
\tilde{u}_{\alpha}^{S} \\
\tilde{u}_{\beta}^{S}
\end{array}\right]=\left[\begin{array}{cc}
Z_{g \alpha \alpha} & Z_{g \alpha \beta} \\
Z_{g \beta \alpha} & Z_{g \beta \beta}
\end{array}\right]\left[\begin{array}{c}
\tilde{i}_{\alpha}^{S} \\
\tilde{i}_{\beta}^{S}
\end{array}\right]
$$

where the superscript ' $L$ ' and ' $S$ ' represent the VSC-side variable and grid-side variable, respectively.

Observing from (37) and (38), there are four channels that can be perturbed, i.e.,

$$
\begin{aligned}
& \tilde{u}_{\alpha}^{L}=\tilde{u}_{\alpha}^{S}+\tilde{u}_{\alpha}^{p t b} \\
& \tilde{u}_{\beta}^{L}=\tilde{u}_{\beta}^{S}+\tilde{u}_{\beta}^{p t b} \\
& \tilde{i}_{\alpha}^{S}=\tilde{i}_{\alpha}^{L}+\tilde{i}_{\alpha}^{p t b} \\
& \tilde{i}_{\beta}^{S}=\tilde{i}_{\beta}^{L}+\tilde{i}_{\beta}^{p t b}
\end{aligned}
$$

where the superscript ' $p t b$ ' denotes the injected perturbation.

Based on (37)-(42), the equivalent block diagram in the $\alpha \beta$ frame is plotted as Fig. 9. According to the definition of loop gain [35], four SISO loop gain models can be obtained by solving the linear system equations (37)-(42), which are given in (43)-(46) shown at the bottom of the page.

$$
\begin{aligned}
& \boldsymbol{Y}_{n}\left(s_{2 X}\right) \tilde{\boldsymbol{u}}_{\alpha \beta}\left(s_{2 X}\right)=\boldsymbol{Y}_{n}\left(s_{2 X}\right)\left[\boldsymbol{Y}_{g \alpha \beta}\left(s_{2 X}\right)-\boldsymbol{Y}_{0}\left(s_{2 X}\right)-\boldsymbol{F}_{P}(P-X)\right]^{-1} \boldsymbol{Y}_{p}\left(s_{2(X-1)}\right) \tilde{\boldsymbol{u}}_{\alpha \beta}\left(s_{2(X-1)}\right) \\
& \boldsymbol{F}_{P}(p)=\boldsymbol{Y}_{n}\left(s_{2(P-p+1)}\right)\left[\boldsymbol{Y}_{g \alpha \beta}\left(s_{2(P-p+1)}\right)-\boldsymbol{Y}_{0}\left(s_{2(P-p+1)}\right)-\boldsymbol{F}_{P}(p-1)\right]^{-1} \boldsymbol{Y}_{p}\left(s_{2(P-p)}\right) \text { with } \quad \boldsymbol{F}_{P}(0)=\mathbf{0}^{2 \times 2} \\
& \boldsymbol{F}_{N}(n)=\boldsymbol{Y}_{p}\left(s_{-2(N-n+1)}\right)\left[\boldsymbol{Y}_{g \alpha \beta}\left(s_{-2(N-n+1)}\right)-\boldsymbol{Y}_{0}\left(s_{-2(N-n+1)}\right)-\boldsymbol{F}_{N}(n-1)\right]^{-1} \boldsymbol{Y}_{n}\left(s_{-2(N-n)}\right) \text { with } \quad \boldsymbol{F}_{N}(0)=\mathbf{0}^{2 \times 2} \\
& T_{\alpha u}=-\left.\frac{\tilde{u}_{\alpha}^{S}}{\tilde{u}_{\alpha}^{L}}\right|_{\tilde{u}_{\beta}^{p h b}=0, \tilde{p}_{\alpha}^{p h b}=0, \tilde{i}_{\beta}^{p h b}=0}=Z_{g \alpha \alpha} Y_{\alpha \alpha}+Z_{g \alpha \beta} Y_{\beta \alpha}-\frac{\left(Z_{g \alpha \alpha} Y_{\alpha \beta}+Z_{g \alpha \beta} Y_{\beta \beta}\right)\left(Z_{g \beta \alpha} Y_{\alpha \alpha}+Z_{g \beta \beta} Y_{\beta \alpha}\right)}{1+Z_{g \beta \alpha} Y_{\alpha \beta}+Z_{g \beta \beta} Y_{\beta \beta}} \\
& T_{\beta u}=-\left.\frac{\tilde{u}_{\beta}^{S}}{\tilde{u}_{\beta}^{L}}\right|_{\tilde{u}_{\alpha}^{p h b}=0, \tilde{p}_{\alpha}^{p h b}=0, \tilde{i}_{\beta}^{p h b}=0}=Z_{g \beta \beta} Y_{\beta \beta}+Z_{g \beta \alpha} Y_{\alpha \beta}-\frac{\left(Z_{g \beta \beta} Y_{\beta \alpha}+Z_{g \beta \alpha} Y_{\alpha \alpha}\right)\left(Z_{g \alpha \beta} Y_{\beta \beta}+Z_{g \alpha \alpha} Y_{\alpha \beta}\right)}{1+Z_{g \alpha \beta} Y_{\beta \alpha}+Z_{g \alpha \alpha} Y_{\alpha \alpha}} \\
& T_{\alpha i}=-\left.\frac{\tilde{i}_{\alpha}^{L}}{\tilde{i}_{\alpha}^{S}}\right|_{\tilde{u}_{\alpha}^{p h b}=0, u_{\beta}^{p h b}=0, \tilde{i}_{\beta}^{p h b}=0}=Z_{g \alpha \alpha} Y_{\alpha \alpha}+Z_{g \beta \alpha} Y_{\alpha \beta}-\frac{\left(Z_{g \alpha \beta} Y_{\alpha \alpha}+Z_{g \beta \beta} Y_{\alpha \beta}\right)\left(Z_{g \alpha \alpha} Y_{\beta \alpha}+Z_{g \beta \alpha} Y_{\beta \beta}\right)}{1+Z_{g \alpha \beta} Y_{\beta \alpha}+Z_{g \beta \beta} Y_{\beta \beta}}
\end{aligned}
$$

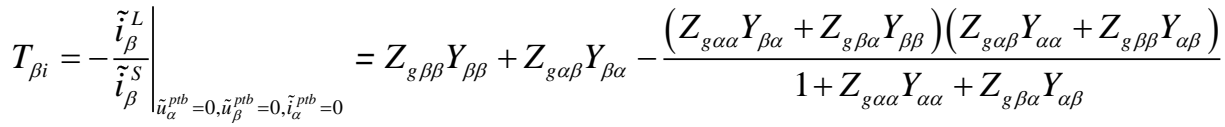


TABLE I

MAIN CIRCUIT AND CONTROL PARAMETERS

\begin{tabular}{cll}
\hline \hline Symbol & \multicolumn{1}{c}{ Description } & \multicolumn{1}{c}{ Value } \\
\hline$u_{g a b c}$ & Rated grid voltage amplitude & $0.62 \mathrm{kV}$ \\
$S_{\text {rated }}$ & Rated power & $2 \mathrm{MVA}$ \\
$\omega_{1}$ & Fundamental angular frequency & $100 \pi \mathrm{rad} / \mathrm{s}$ \\
$u_{d c}$ & DC-link voltage & $1200 \mathrm{~V}$ \\
$L_{f} / R_{f}$ & Input filter inductance/resistance & $0.4 \mathrm{mH} / 0 \Omega$ \\
$L_{g} / R_{g}$ & Grid inductance/resistance & $0.5 \mathrm{mH} / 0 \Omega$ \\
$\omega_{i} / \omega_{v}$ & ADC cutoff frequency & $2000 \pi \mathrm{rad} / \mathrm{s}$ \\
$\xi$ & Damping ratio of the SOGI-QSG & 0.707 \\
$k_{p p}$ & Proportional gain of the PLL & 0.2 \\
$k_{i p}$ & Integral gain of the PLL & 903 \\
$k_{p c}$ & Proportional gain of the current regulator & 0.64 \\
$k_{r c}$ & Resonant gain of the current regulator & 210 \\
$f_{s}$ & Switching frequency & $2.5 \mathrm{kHz}$ \\
\hline
\end{tabular}

Although the obtained loop gain models $T_{\alpha u}, T_{\beta u}, T_{\alpha i}$, and $T_{\beta i}$ have different expressions, they are the same on the stability assessment, whose proof is given in Appendix-B. Thus, any loop gain can be used for stability analysis. In the following, only the $T_{\alpha u}$ is considered for simplicity.

The merits of the developed SISO loop gain models are summarized as:

1) The loop gain directly reflects the stability margin information of the system.

2) The loop gain model includes the coupling effects of cross-channel and grid impedance.

3) The system stability can be assessed by applying the Nyquist criterion to $T_{x}(x=\alpha u, \beta u, \alpha i$, and $\beta i)$.

4) The traditional SISO perturbation and measurement scheme becomes a viable solution for three-phase systems.

Remark: In [10], the equivalent SISO converter/grid impedance models are separately calculated by

$$
\begin{gathered}
Z_{\alpha}^{L}=-\left.\frac{\tilde{u}_{\alpha}^{L}}{\tilde{i}_{\alpha}^{L}}\right|_{\tilde{u}_{\beta}^{p t b}=0, \tilde{i}_{\alpha}^{p t b}=0, \tilde{i}_{\beta}^{p t b}=0} \\
Z_{\alpha}^{S}=\left.\frac{\tilde{u}_{\alpha}^{S}}{\tilde{i}_{\alpha}^{S}}\right|_{\tilde{u}_{\beta}^{p t b}=0, \tilde{i}_{\alpha}^{p t b}=0, \tilde{i}_{\beta}^{p t b}=0}
\end{gathered}
$$

It is observed that $T_{\alpha u}=Z_{\alpha}{ }^{S} / Z_{\alpha}{ }^{L}\left(\tilde{i}_{\alpha}{ }^{L}=\tilde{i}_{\alpha}{ }^{S}\right)$. To obtain a more compact model, [33] defines the loop impedance $Z_{\alpha}{ }^{L O O P}=-\tilde{u}_{\alpha}{ }^{p t b} /$ $\tilde{i}_{\alpha}{ }^{L}=Z_{\alpha}{ }^{S}+Z_{\alpha}{ }^{L}$, which is the closed-loop characteristic equation. Clearly, all three models are identical for stability assessment.

From the perspective of measurement, the equivalent SISO converter/grid impedances $\left(Z_{\alpha}{ }^{S}\right.$ and $\left.Z_{\alpha}{ }^{L}\right)$ need to be separately measured. In contrast, the loop impedance $Z_{\alpha}{ }^{L O O P}$ and the proposed loop gain $T_{\alpha u}$ both can be obtained by a single measurement test, reducing the measurement complexity and information redundancy. However, most stability criteria (e.g., Nyquist stability criterion) are designed for the open-loop transfer function (i.e., loop gain), which limits the application of loop impedance for stability analysis. In addition, the loop gain can directly reflect the stability margin information.

In summary, the use of the loop gain concept in system modeling is promising due to its simplicity and convenience for physical interpretation.
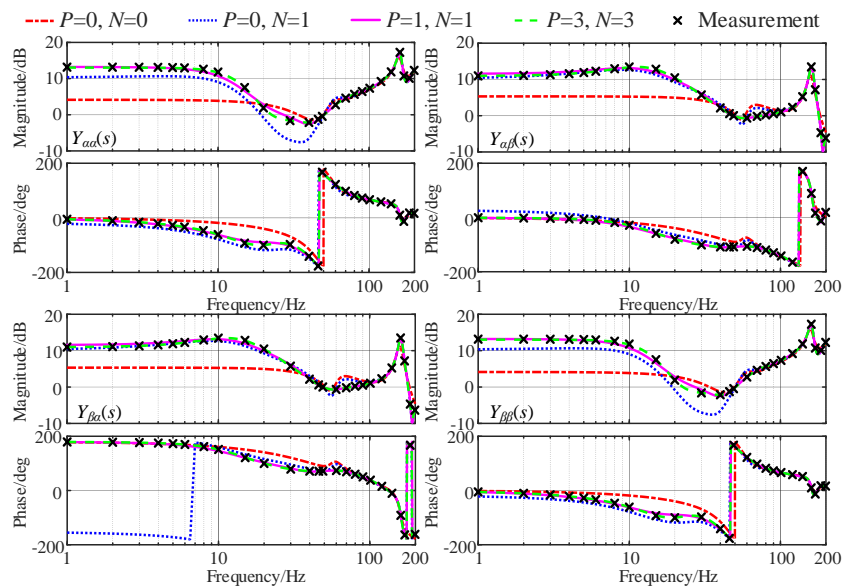

Fig. 10. Comparative analysis of the RAM $Y(s)$ with different truncation orders.
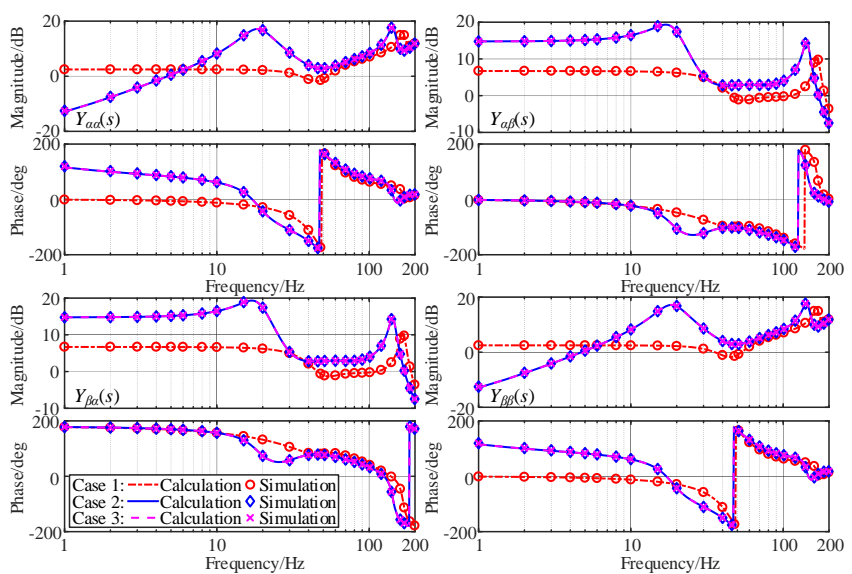

Fig. 11. Frequency responses of $\boldsymbol{Y}(s)$ under Case 1-3. Lines: Model prediction; Dots: Simulation results.

\section{Simulation VALIDATION}

In order to verify the proposed models, a time-domain simulation model is built in Matlab/Simulink according to the three-phase grid-tied VSC structure in Fig. 1. The system parameters are provided in Table I.

\section{A. Frequency Scan Validation}

The model accuracy depends on the chosen truncation order. When finite-order truncation is performed, frequency response deviation may occur. In Fig. 10, under $u_{g a}=u_{g b}=u_{g c}=1$ p.u. and $L_{g a} / R_{g a}=0$ p.u., $L_{g b} / R_{g b}=1$ p.u., $L_{g c} / R_{g c}=2$ p.u., the RAM $\boldsymbol{Y}(s)$ with different truncation orders are compared. The selection of truncation order mainly influences the frequency response of $\boldsymbol{Y}(s)$ below the fundamental frequency. Moreover, the frequency response deviation decreases as the truncation order increases. An explicit formula for truncation order selection has not been given yet. As a result, the truncation parameters $P$ and $N$ are usually evaluated iteratively. In the work presented in the following, $N=3$ and $P=3$ have been chosen.

Under the grid impedances of $L_{g a} / R_{g a}=L_{g b} / R_{g b}=L_{g c} / R_{g c}=0.5$ p.u., three different grid voltage conditions are tested to illustrate the impact of grid voltage imbalances:

1) Case 1: $u_{g a}=u_{g b}=u_{g c}=1$ p.u. ( $V_{p}=0.96$ p.u., $V_{n}=0$ p.u. $)$;

2) Case 2: $u_{g a}=u_{g b}=u_{g c}=0.66$ p.u. ( $V_{p}=0.6$ p.u., $V_{n}=0$ p.u. $)$;

3) Case 3: $u_{g a}=u_{g b}=1$ p.u., $u_{g c}=0$ p.u. ( $V_{p}=0.6$ p.u., $V_{n}=0.33$ p.u.). 


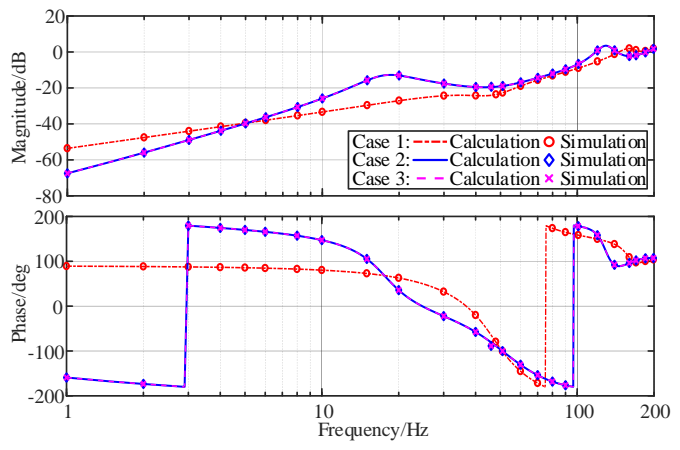

Fig. 12. Frequency responses of $T_{\text {ou }}(s)$ under Case 1-3. Lines: Model prediction; Dots: Simulation results.
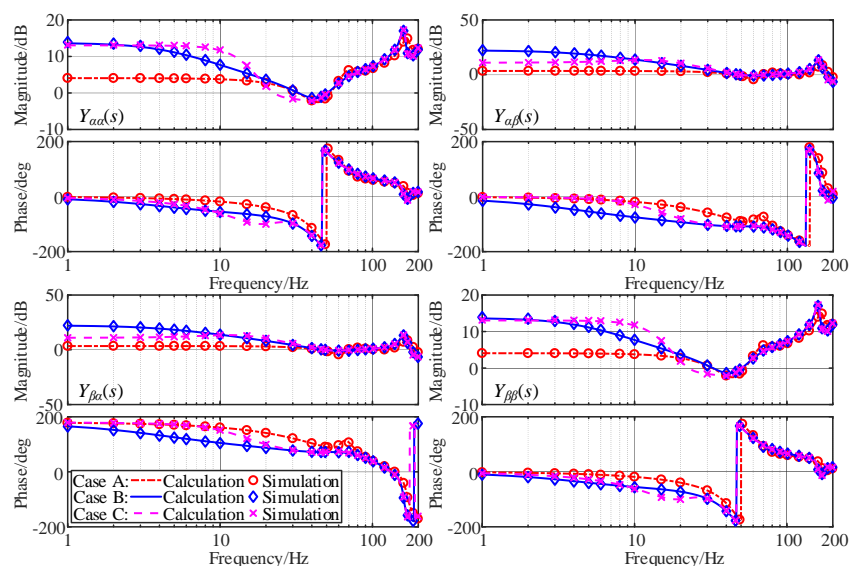

Fig. 13. Frequency responses of $\boldsymbol{Y}(s)$ under Case A-C. Lines: Model prediction; Dots: Simulation results.

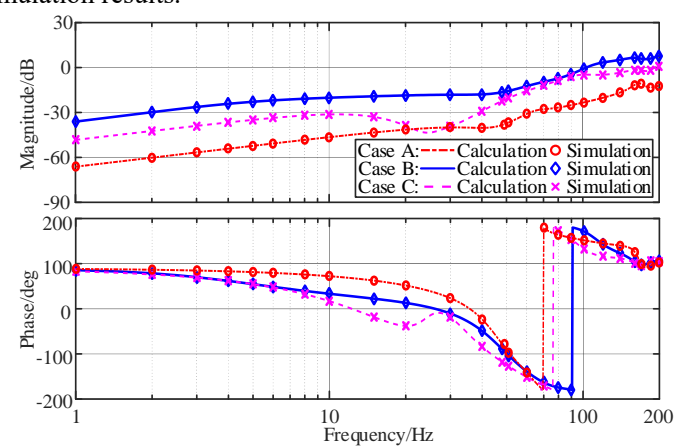

Fig. 14. Frequency responses of $T_{a u}(s)$ under Case A-C. Lines: Model prediction; Dots: Simulation results.

The resulting frequency response curves for Case 1-3 are shown in Fig. 11 for the RAM model $\boldsymbol{Y}(s)$ and in Fig. 12 for the loop gain model $T_{\alpha u}$.

To analyze the converter-grid coupling effect, the following three cases are considered in the frequency scanning:

1) Case A: $L_{g a} / R_{g a}=L_{g b} / R_{g b}=L_{g c} / R_{g c}=0.1$ p.u. $\left(V_{p}=1\right.$ p.u., $V_{n}=0$ p.u.);

2) Case B: $L_{g a} / R_{g a}=L_{g b} / R_{g b}=L_{g c} / R_{g c}=1$ p.u. $\left(V_{p}=0.84\right.$ p.u., $V_{n}=0$ p.u.);

3) Case C: $L_{g a} / R_{g a}=0$ p.u., $L_{g b} / R_{g b}=1$ p.u., $L_{g c} / R_{g c}=2$ p.u. ( $V_{p}=0.84$ p.u., $V_{n}=0.31$ p.u.).

The resulting frequency response curves for Case A-C are shown in Fig. 13 for the RAM model $\boldsymbol{Y}(s)$ and in Fig. 14 for the loop gain model $T_{\alpha u}$ under the balanced grid voltages ( $u_{g a}=u_{g b}=u_{g c}=1$ p.u.).

The following observations support the claims in the previous sections:

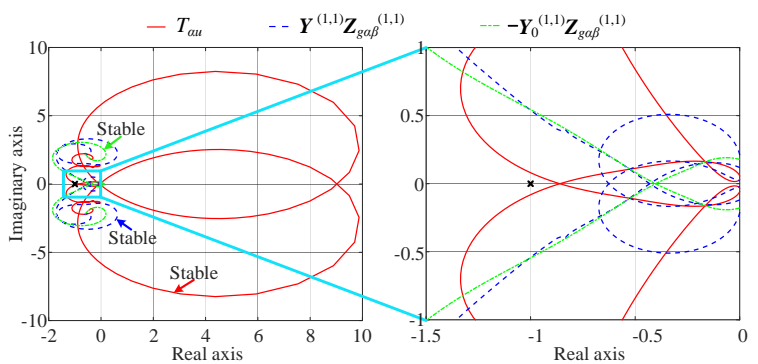

(a) $m=2.3$

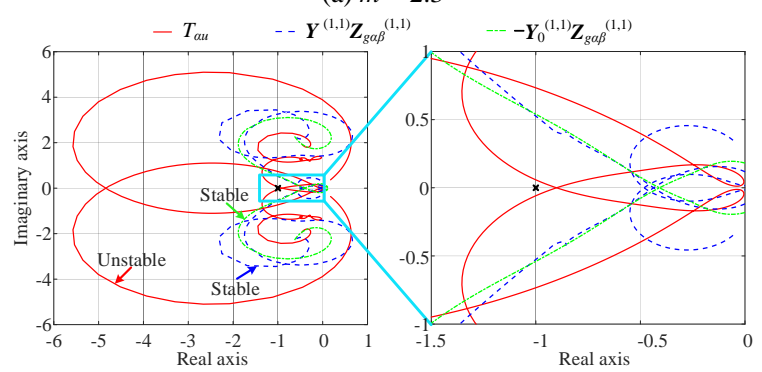

(b) $m=2.4$

Fig. 15. Nyquist diagrams for the loop gains under different $m$.

1) The two established models match the measured results in all cases. This validates the accuracy of the proposed modeling approach.

2) Fig. 11 shows that the converter admittance is decoupled with the negative-sequence voltage $V_{n}$ due to the use of DSOGIPLL.

3) From Fig. 13, it is observed that the converter admittance varies along with the grid impedance, which is mainly in the low-frequency regions.

4) Although both the unbalanced grid voltages and asymmetric grid impedances lead to the PCC voltage imbalance, they have different effects on the VSC admittance.

\section{B. Stability Analysis Against Various Grid Impedances}

To analyze the effect of asymmetric grid impedances on system stability, $Z_{g c}$ is taken as an adjustable parameter, i.e., $Z_{g a}=Z_{g b}=0.7$ p.u. and $Z_{g c}=m$ p.u.. The value of $m$ denotes the asymmetrical factor of grid impedance. In what follows, under the grid voltage conditions of $u_{g a}=u_{g b}=u_{g c}=1$ p.u., two asymmetric factors: $m=2.3$ and $m=2.4$ are considered for stability assessment.

Fig. 15(a)-(b) shows the Nyquist diagrams of the loop gain $T_{a u}$ and the reduced-order impedance ratios $\boldsymbol{Y}^{(1,1)} \boldsymbol{Z}_{g \alpha \beta}{ }^{(1,1)}$ and $\boldsymbol{Y}_{0}^{(1,1)} \boldsymbol{Z}_{g \alpha \beta^{(1,1)}}$. It can be found that the stability performance degrades with the increase of $m$. Specifically, when $m=2.4$, a conflicting stability conclusion is arrived at. The system is predicted to be unstable by $T_{a u}$, whereas it is deemed to be stable by $\boldsymbol{Y}^{(1,1)} \boldsymbol{Z}_{g \alpha \beta^{(1,1)}}$ and $-\boldsymbol{Y}_{0}^{(1,1)} \boldsymbol{Z}_{g \alpha \beta^{(1,1)}}$.

To further identify where the harmonic frequencies are located, the Nyquist diagram of $T_{\alpha u}$ under $m=2.4$ is plotted in Fig. 16. It is observed that a negative amplitude margin (GM=13.6 $\mathrm{dB})$ and a negative phase margin $\left(\mathrm{PM}=-37.5^{\circ}\right)$ are respectively identified at the frequencies $13.3 \mathrm{~Hz}$ and $14.4 \mathrm{~Hz}$, indicating an unstable VSC-grid system [36]. In addition, the harmonic frequency $f_{c}$ is expected to be around $14 \mathrm{~Hz}$. Due to the FCE, it is emphasized that other sideband components will also appear with harmonic frequency shifting proportional to the double fundamental frequency $\left(f_{c} \pm 2 k f_{1}, f_{1}=\omega_{1} /(2 \pi), k \in \mathbb{Z}\right)$. 


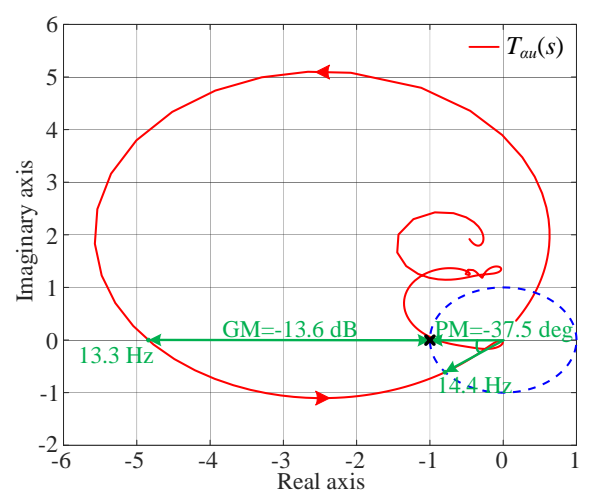

Fig. 16. Nyquist diagram of $T_{a u}(s)$ under $m=2.4$.

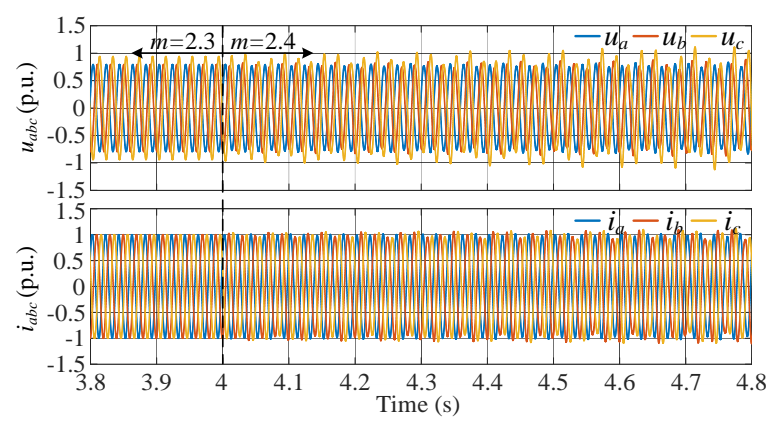

(a) Time-domain waveforms

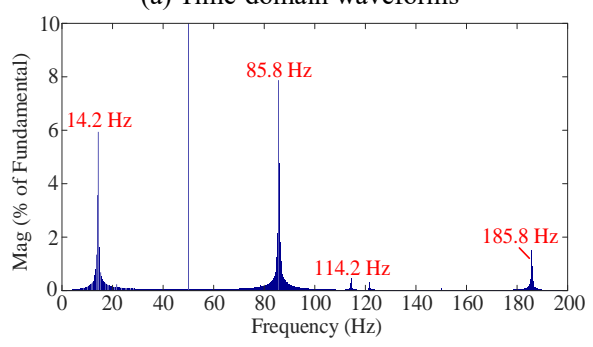

(b) Spectrum of $i_{a}$

Fig. 17. Simulation results with $m=2.3$ changed to $m=2.4$ at $4 \mathrm{~s}$.

Fig. 17 shows a time-domain simulation where $m$ is changed from 2.3 to 2.4 at $4 \mathrm{~s}$ to complement the stability analysis. As observed, the PCC voltages $u_{a b c}$ are unbalanced, which is caused by the asymmetric grid impedances $Z_{g a b c}$. Moreover, oscillations can be found in PCC voltages $u_{a b c}$ and grid currents $i_{a b c}$ when $m=2.4$. By performing a Fourier analysis on the phase current $i_{a}$, the oscillation frequencies of $14.2 \mathrm{~Hz}$ and the coupled frequencies of $85.8 \mathrm{~Hz}, 114.2 \mathrm{~Hz}$, and $185.8 \mathrm{~Hz}$ are all identified as shown in Fig. 17(b), which closely agrees with the analyzed results in Fig. 16.

Based on the above analysis and validation, it is known that the reduced-order impedance models are over-optimistic on the stability assessment, while the loop gain model gives accurate results. As a result, an accurate stability analysis must take all the frequency coupling components into account.

\section{CONCLUSION}

In this paper, a unified SISO loop gain modeling for threephase VSCs under both symmetric and asymmetric ac grids is proposed, which takes account of the FCE. It is illustrated that the two-by-two RAM model and SISO loop gain model can accurately characterize the frequency coupling dynamics of arbitrary order without large-dimensional matrix operation. The concept of admittance is extended by RAM. The RAM depends not only on the VSC itself but also on the grid admittance. Different choices of the perturbation injection point lead to different loop gains, and they are identical on the stability assessment. The stability analysis can be simplified by applying the classic SISO stability criteria (e.g., Nyquist criterion) to the loop gain model. In addition, the loop gain allows the traditional SISO perturbation and measurement scheme to be used for detecting the stability margin information of the system. The use of the loop gain concept can greatly simplify measurementbased analysis where tedious modeling is not necessary.

\section{APPENDIX}

\section{A. Derivation of grid admittance model $\boldsymbol{Y}_{g \alpha \beta}(s)$}

In this study, a general grid impedance condition is considered that $Y_{g a}(s)=s L_{g a}+R_{g a}, \quad Y_{g b}(s)=s L_{g b}+R_{g b}, \quad$ and $Y_{g c}(s)=s L_{g c}+R_{g c}$ are not necessarily the same. In the following, how to derive $\boldsymbol{Y}_{g \alpha \beta}(s)$ is briefly introduced.

In the $a b c$-frame, the three-phase admittance model is

$$
\left[\begin{array}{l}
\tilde{i}_{a} \\
\tilde{i}_{b} \\
\tilde{i}_{c}
\end{array}\right]=\left[\begin{array}{ccc}
Y_{g a} & 0 & 0 \\
0 & Y_{g b} & 0 \\
0 & 0 & Y_{g c}
\end{array}\right]\left[\begin{array}{l}
\tilde{u}_{a} \\
\tilde{u}_{b} \\
\tilde{u}_{c}
\end{array}\right]
$$

where Laplace variable $s$ is omitted for brevity.

The normalized-Clarke transformation is applied firstly, the voltage vector and current vector can be translated from the $a b c$ - to the $\alpha \beta \gamma$-reference frames as

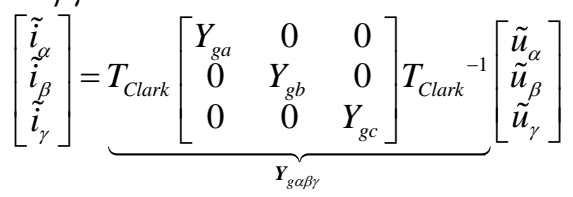

where

$$
T_{\text {Clark }}=\frac{2}{3}\left[\begin{array}{ccc}
1 & -\frac{1}{2} & -\frac{1}{2} \\
0 & \frac{\sqrt{3}}{2} & -\frac{\sqrt{3}}{2} \\
\frac{1}{2} & \frac{1}{2} & \frac{1}{2}
\end{array}\right]
$$

Denoting $\boldsymbol{Y}_{g a \beta \gamma}$ as

$$
\boldsymbol{Y}_{g \alpha \beta \gamma}=\left[\begin{array}{lll}
Y_{11} & Y_{12} & Y_{13} \\
Y_{21} & Y_{22} & Y_{23} \\
Y_{31} & Y_{32} & Y_{33}
\end{array}\right]
$$

where the detailed expressions of the entries $Y_{x y}(x=1,2,3 ; y=1$, 2,3) in $\boldsymbol{Y}_{g \alpha \beta \gamma}$ can be easily solved from (A2).

Due to the three-wire structure of the studied VSC system, $\tilde{i}_{\gamma}=0$ always holds so that

$$
\tilde{u}_{\gamma}=-\frac{Y_{31}}{Y_{33}} \tilde{u}_{\alpha}-\frac{Y_{32}}{Y_{33}} \tilde{u}_{\beta}
$$

By substituting (A5) into (A2), it is derived that

$$
\left[\begin{array}{c}
\tilde{i}_{\alpha} \\
\tilde{i}_{\beta}
\end{array}\right]=\underbrace{\left[\begin{array}{cc}
Y_{11}-\frac{Y_{13} Y_{31}}{Y_{33}} & Y_{12}-\frac{Y_{13} Y_{32}}{Y_{33}} \\
Y_{21}-\frac{Y_{23} Y_{31}}{Y_{33}} & Y_{22}-\frac{Y_{23} Y_{32}}{Y_{33}}
\end{array}\right]}_{Y_{\beta \alpha \beta}}\left[\begin{array}{l}
\tilde{u}_{\alpha} \\
\tilde{u}_{\beta}
\end{array}\right]
$$

Comparing (A6) and (28), $Y_{g \alpha \alpha}, Y_{g \alpha \beta}, Y_{g \beta \alpha}$, and $Y_{g \beta \beta}$ are obtained. 


\section{B. Proof of the identical stability assessment of different SISO loop gain models}

According to the definition of loop gain, the system stability is assessed by the characteristic equations [35], which are consistently expressed as

$$
1+T_{x}=0, \quad(x=\alpha u, \beta u, \alpha i, \beta i)
$$

Substituting (43)-(46) into (B1) and performing simple mathematical manipulations, it is not hard to obtain that the four characteristic equations share the same solution, which is given by

$$
\begin{aligned}
& 1+Z_{g \beta \alpha} Y_{\alpha \beta}+Z_{g \beta \beta} Y_{\beta \beta}+Z_{g \alpha \alpha} Y_{\alpha \alpha}+Z_{g \alpha \beta} Y_{\beta \alpha} \\
& +\left(Z_{g \alpha \alpha} Z_{g \beta \beta}-Z_{g \alpha \beta} Z_{g \beta \alpha}\right)\left(Y_{\alpha \alpha} Y_{\beta \beta}-Y_{\alpha \beta} Y_{\beta \alpha}\right)=0
\end{aligned}
$$

The system stability is determined by the roots of (B2) [35]. Therefore, $T_{a u}, T_{\beta u}, T_{a i}$, and $T_{\beta i}$ are the same in terms of stability.

\section{REFERENCES}

[1] F. Blaabjerg, Y. Yang, D. Yang, and X. Wang, "Distributed powergeneration systems and protection," Proc. IEEE, vol. 105, no. 7, pp. 1311-1331, Jul. 2017.

[2] L. Zhang, L. Harnefors, and H.-P. Nee, "Modeling and control of VSCHVDC links connected to island systems," IEEE Trans. Power Syst., vol. 26, no. 2, pp. 783-793, May 2011

[3] J. Sun, "Small-signal methods for AC distributed power systems-A review," IEEE Trans. Power Electron., vol. 24, no. 11, pp. 2545-2554, Nov. 2009.

[4] X. Wang and F. Blaabjerg, "Harmonic stability in power electronicbased power systems: concept, modeling, and analysis," IEEE Trans. Smart Grid, vol. 10, no. 3, pp. 2858-2870, May 2019.

[5] X. Yue, X. Wang, and F. Blaabjerg, "Review of small-signal modeling methods including frequency-coupling dynamics of power converters," IEEE Trans. Power Electron., vol. 34, no. 4, pp. 3313-3328, Apr. 2019.

[6] S. Hiti, D. Boroyevich, and C. Cuadros, "Small-signal modeling and control of three-phase PWM converters," in IEEE Ind. Appl. Soc. Annu. Meeting, pp. 1143-1150, Oct. 1994.

[7] M. Belkhayat, "Stability criteria for AC power systems with regulated loads," Ph.D. dissertation, Purdue Univ., West Lafayette, IN, Dec. 1997.

[8] L. Harnefors, M. Bongiorno and S. Lundberg, "Input-admittance calculation and shaping for controlled voltage-source converters," IEEE Trans. Ind. Electron., vol. 54, no. 6, pp. 3323-3334, Dec. 2007.

[9] B. Wen, D. Boroyevich, R. Burgos, P. Mattavelli and Z. Shen, "Analysis of d-q small-signal impedance of grid-tied inverters," IEEE Trans. Power Electron., vol. 31, no. 1, pp. 675-687, Jan. 2016.

[10] A. Rygg, M. Molinas, C. Zhang, and X. Cai, "A modified sequence domain impedance definition and its equivalence to the dq-domain impedance definition for the stability analysis of AC power electronic system," IEEE J. Emerg. Sel. Topics Power Electron., vol. 4, no. 4, pp. 1383-1396, Dec. 2016.

[11] S. Shah and L. Parsa, "Impedance modeling of three-phase voltage source converters in dq, sequence, and phasor domains," IEEE Trans. Energ. Conv., vol. 32, no. 3, pp. 1139-1150, Sep. 2017.

[12] X. Wang, L. Harnefors and F. Blaabjerg, "Unified impedance model of grid-connected voltage-source converters," IEEE Trans. Power Electron., vol. 33, no. 2, pp. 1775-1787, Feb. 2018.

[13] N. M. Wereley, "Analysis and control of linear periodically time varying systems," Ph.D. dissertation, Dept. of Aeronautics and Astronautics, MIT Inst. Technol., 1991

[14] E. Mollerstedt and B. Bernhardsson, "Out of control because of harmonics-an analysis of the harmonic response of an inverter locomotive," IEEE Control Syst. Mag., vol. 20, no. 4, pp. 70-81, Aug. 2000.

[15] S. R. Sanders, J. M. Noworolski, X. Z. Liu and G. C. Verghese, "Generalized averaging method for power conversion circuits," IEEE Trans. Power Electron., vol. 6, no. 2, pp. 251-259, Apr. 1991.

[16] A. M. Stankovic and T. Aydin, "Analysis of asymmetrical faults in power systems using dynamic phasors," IEEE Trans. Power Syst., vol. 15, no. 3, pp. 1062-1068, Aug. 2000.
[17] D. Maksimovic, A. M. Stankovic, V. J. Thottuvelil, and G. C. Verghese, "Modeling and simulation of power electronic converters," Proc. IEEE, vol. 89, no. 6, pp. 898-912, Jun. 2001.

[18] O. C. Sakini and J. Beerten, "Equivalent multiple dq-frame model of the MMC using dynamic phasor theory in the $\alpha \beta \mathrm{z}$-frame," IEEE Trans. Power Del., vol. 35, no. 6, pp. 2916-2927, Dec. 2020.

[19] J. Sun and K. Karimi, "Small-signal input impedance modeling of line frequency rectifiers," IEEE Trans. Aerosp. Electron. Syst., vol. 44, no. 4, pp. 1489-1497, Oct. 2008.

[20] M. K. Bakhshizadeh, X. Wang, F. Blaabjerg, J. Hjerrild, L. Kocewiak, C. L. Bak and B. Hesselbak, "Couplings in phase domain impedance modeling of grid-connected converters," IEEE Trans. Power Electron., vol. 31, no. 10, pp. 6792-6796, Oct. 2016.

[21] K. Shi, Y. Wang, Y. Sun, P. Xu, and, F. Gao, "Frequency-coupled impedance modeling of virtual synchronous generator," IEEE Trans. Power Syst., vol. 36, no. 4, pp. 3692-3700, Jul. 2021.

[22] J. Sun and H. Liu, "Sequence impedance modeling of modular multilevel converters," IEEE J. Emerg. Sel. Topics Power Electron., vol. 5, no. 4, pp. 1427-1443, Dec. 2017

[23] S. Shah, P. Koralewicz, V. Gevorgian, and L. Parsa, "Small-signal modeling and design of phase-locked loops using harmonic signal-flow graphs," IEEE Trans. Energ. Conv., vol. 35, no. 2, pp. 600-610, Jun. 2020.

[24] E. Mollerstedt, "Dynamic analysis of harmonics in electrical systems," Ph.D. dissertation, Dept. Automatic Control, Lund Inst. Technol., Lund, Sweden, 2000.

[25] H. Nian, L. Chen, Y. Xu, H. Huang, and J. Ma, "Sequences domain impedance modeling of three-phase grid-connected converter using harmonic transfer matrices," IEEE Trans. Energ. Conv., vol. 33, no. 2, pp. 627-638, Jun. 2018.

[26] J. Lin, M. Su, Y. Sun, D. Yang, and S. Xie, "Recursive SISO impedance modeling of single-phase voltage source rectifiers," IEEE Trans. Power Electron., early access, 2021.

[27] A. M. Stankovic, S. R. Sanders, and T, Aydin, "Dynamic phasors in modeling and analysis of unbalanced polyphase ac machines," IEEE Trans. Energ. Conv., vol. 17, no. 1, pp. 107-113, Mar. 2002.

[28] P. C. Stefanov and A. M. Stankovic, "Modeling of UPFC operation under unbalanced conditions with dynamic phasors," IEEE Trans. Power Syst., vol. 17, no. 2, pp. 395-403, May 2002.

[29] M. Cespedes and J. Sun, "Methods for stability analysis of unbalanced three-phase systems," in Proc. IEEE Energ. Conv. Congr. Expo. (ECCE), pp. 3090-3097, 2012.

[30] Y. Cen, M. Huang, and X. Zha, "Modeling method of sequence admittance for three-phase voltage source converter under unbalanced grid condition," J. Mod. Power Syst. Clean Energ., vol. 6, pp. 595-606, Mar. 2018

[31] C. Zhang, M. Molinas, A. Rygg, J. Lyu and X. Cai, "Harmonic transfer function-based impedance modeling of a three-phase VSC for asymmetric AC grids stability analysis," IEEE Trans. Power Electron., vol. 34, no.12, pp. 12552-12566, Dec. 2019.

[32] Y. Liao, X. Wang, X. Yue, and L. Harnefors, "Complex-valued multifrequency admittance model of three-phase VSCs in unbalanced grids," IEEE J. Emerg. Sel. Topics Power Electron., vol. 8, no.12, pp. 1934-1946, Jun. 2020.

[33] C. Zhang, X. Cai, A. Rygg, and M. Molinas, "Sequence domain SISO equivalent models of a grid-tied voltage source converter system for small-signal stability analysis," IEEE Trans. Energ. Conv., vol. 33, no. 2, pp. 741-749, Jun. 2018.

[34] P. Rodriguez, R. Teodorescu, I. Candela, A. V. Timbus, M. Liserre, and F. Blaabjerg, "New positive-sequence voltage detector for grid synchronization of power converters under faulty grid conditions," IEEE Power Electron. Specialists Conf. (APEC), pp. 1-7, 2006.

[35] R. Erickson and D. Maksimovic, Fundamentals of Power Electronics, 2nd ed. Norwell, M.A., Kluwer, 2004.

[36] C. M. Wildrick, F. C. Lee, B. H. Cho, and B. Choi, "A method of defining the load impedance specification for a stable distributed power system," IEEE Trans. Power Electron., vol. 10, no. 3, pp. 280-285, May 1995.

[37] J. Lin, M. Su, Y. Sun, X. Li, S. Xie, G. Zhang, F. Blaabjerg, and J. Feng, "Accurate loop gain modeling of digitally controlled Buck converters," IEEE Trans. Ind. Electron., early access, 2021. 